\title{
WHAT CAN THE COSMIC MICROWAVE BACKGROUND TELL US ABOUT THE OUTER SOLAR SYSTEM?
}

\author{
Daniel Babich, ${ }^{1,2}$ Cullen H. Blake, ${ }^{1,3}$ and Charles L. Steinhardt ${ }^{1}$ \\ Received 2006 October 31; accepted 2007 May 4
}

\begin{abstract}
We discuss two new observational techniques that use observations of the cosmic microwave background (CMB) to place constraints on the mass, distance, and size distribution of small objects in the Kuiper Belt and inner Oort Cloud, collectively known as trans-Neptunian objects (TNOs). The first new technique considers the spectral distortion of the isotropic, or monopole, CMB by TNOs that have been heated by solar radiation to temperatures above that of the CMB. We apply this technique to the spectral measurements of the CMB by the Far Infrared Absolute Spectrophotometer on the Cosmic Background Explorer. The second technique utilizes the change in amplitude of the TNO signal due to the orbital motion of the observer to separate the TNO signal from the invariant extragalactic CMB and construct a map of the mass distribution in the outer solar system. We estimate the ability of future CMB experiments to create such a map.
\end{abstract}

Subject headings: cosmic microwave background - Kuiper Belt — Oort Cloud

Online material: color figures

\section{INTRODUCTION}

While we know that in some manner dynamical instabilities in the protoplanetary disk led to the formation of planetesimals and planets, the precise details of this process are not well understood (Goldreich et al. 2004; Lissauer \& Stevenson 2007). Luckily, just as nature has provided us with the cosmic microwave background $(\mathrm{CMB})$ as a relic of the early universe, there are similar relics of this chaotic period of formation. Objects in the outer solar system, collectively known as trans-Neptunian objects (TNOs), with semimajor axes $40 \mathrm{AU} \leq a \leq 10^{5} \mathrm{AU}$ are believed to provide clues that may help us understand the early history of the solar system. The number density and mass distribution of TNOs contain valuable information about the properties of the protoplanetary disk out of which the solar system formed, and the dynamical properties of these TNOs can constrain migratory motion of the planets. We show in this paper that the $\mathrm{CMB}$ itself may be the key to unlocking the mysteries of these objects.

To date, the entirety of our observational evidence about the structure of the solar system beyond Neptune consists of observations of comets that have been perturbed into the inner solar system and of relatively large, nearby objects detected through their reflected sunlight (Bernstein et al. 2004). Because of the strong dependence of the reflected sunlight on the object's distance and radius $\left(\propto D^{-4}\right.$ and $\left.\propto R^{-2}\right)$, it is very difficult to find objects smaller or more distant than, for example, (90377) Sedna (Brown et al. 2004), a body $1180-1800 \mathrm{~km}$ in diameter detected at $90 \mathrm{AU}$, or $2003 \mathrm{UB}_{313}$ (Brown et al. 2005), a body $2400 \mathrm{~km}$ in diameter detected near its aphelion distance of $96 \mathrm{AU}$.

Long-period comets (LPCs) associated with the Oort Cloud originate in its outermost reaches, because objects with large semimajor axes can be preferentially ejected into the inner solar system. ${ }^{4}$

\footnotetext{
1 Harvard-Smithsonian Center for Astrophysics, 60 Garden Street, Cambridge, MA 02138; dbabich@cfa.harvard.edu,cblake@cfa.harvard.edu, csteinha@cfa .harvard.edu.

2 California Institute of Technology, Theoretical Astrophysics, MC 130-33, Pasadena, CA 91125.

3 Harvard Origins of Life Initiative Fellow.

4 Perturbations by Jupiter are large enough to either eject the new LPC on a hyperbolic orbit or keep it within 1000 AU of the Sun. This originally led Oort to postulate that comets on orbits with large aphelia are always entering the inner solar system for the first time.
}

The observed aphelia of LPCs place the outer limits of the Oort Cloud at a distance of approximately 25,000 AU (Oort 1950; Marsden \& Sekanina 1971), while simulations of the Oort Cloud's formation, assuming that the Sun formed in a star cluster, suggest that a significant amount of mass may lie in an inner Oort Cloud, which is located at $1000 \mathrm{AU}$ (Hills 1981; Fernandez 1997; Dones et al. 2004). To this point, we have no methods capable of detecting smaller objects in the Kuiper Belt or any objects in the inner Oort Cloud.

In this paper we develop two new methods capable of exploring the distribution of TNOs. Both utilize the CMB as a standard spectral template, namely, a blackbody with a given temperature emanating from a surface at infinity. The Sun heats Kuiper Belt and Oort Cloud objects to a temperature above that of the CMB. These objects extinguish part of the CMB and emit blackbody radiation, inducing a spectral distortion in the observed CMB. By constraining these spectral distortions we can constrain the radial and mass distribution of TNOs.

Some similar topics have previously been discussed. Detections of zodiacal light, both through its reflected optical sunlight and its emitted infrared radiation, have led to detailed models of interplanetary dust in the solar system (Fixsen \& Dwek 2002). This dust is believed to come from the comae of comets and the collisional debris of asteroids. The infrared emission of Kuiper Belt objects has been employed in a similar fashion to this paper in order to constrain the Kuiper Belt (Kenyon \& Windhorst 2001; Teplitz et al. 1999; Backman et al. 1995). The main advantage of using the CMB as the source of background radiation is that its properties are much better understood and more uniform than the far-infrared background, allowing for a greater precision. In addition, the longer relevant wavelengths of the CMB spectrum enable us to apply the same test to the inner Oort Cloud, which is at too low of a temperature to emit any substantial amount of radiation in the infrared bands.

In $\S 2$ we calculate the blackbody radiation emitted by objects in the outer solar system including a discussion of the temperature of distant objects. In $\S 3$ we use the Far Infrared Absolute Spectrophotometer (FIRAS) to place constraints on the total mass and size distribution of the smallest objects in the Kuiper belt. In $\S 4$ we propose a new method of using WMAP to develop a twodimensional map of the smallest objects in the Kuiper Belt, 
holding out the possibility that for sufficient mass we may be able to determine the distance in addition to the mass and direction.

\section{OUTER SOLAR SYSTEM SPECTRAL DISTORTIONS}

The extremely high interaction rates between matter and radiation during the early universe cause the primordial CMB spectrum to have a blackbody distribution to incredibly high precision (Mather et al. 1991; Peebles 1993). However, as the universe expands and these interaction rates decrease, new sources of energy injection can distort the CMB spectrum and photon-baryon plasma is now incapable of producing a blackbody spectrum again. In addition to energy injection at high redshifts, there are several low-redshift processes that are capable of distorting the CMB spectrum (see Tegmark \& Silk [1994] and Fixsen \& Kogut [2002] for an overview). In this paper we try to constrain the properties of TNOs from the absence of spectral distortions, so any additional process that may produce these distortions does not affect our ability to place these constraints on the outer solar system, but in principle strengthens our conclusions. We consequently ignore these other effects in this paper.

It should be emphasized that these spectral distortions are not the same as the chemical potential $(\mu)$ or Compton $y$-distortions often discussed in the literature (Tegmark \& Silk 1994; Fixsen et al. 1997). Of course, there is a level of degeneracy between the spectral distortion considered in this paper, namely a weighted sum of blackbodies, and the chemical potential and Compton $y$-distortions, so our signal will result in a nonzero chemical potential and Compton $y$-distortion if either of these models was assumed in the analysis.

\subsection{Calculation}

We are interested in the aggregate blackbody emission from objects at a given distance in the outer solar system. The distorted CMB intensity ${ }^{5}$ spectrum due to these objects will be

$$
\delta I_{\nu}=\tau\left[B_{\nu}\left(T_{\mathrm{TNO}}\right)-B_{\nu}\left(T_{\mathrm{CMB}}\right)\right],
$$

where the optical depth, or equivalently the geometric covering fraction since we are working in the geometric optics limit, is expressed as

$$
\tau=\int \frac{\pi R^{2}}{4 \pi D^{2}} n(M) d M
$$

where $R$ is the radius of the object and $D$ is the approximately identical distance to the TNO from both the Sun and the observer. These two distances are not exactly equal, and the TNO-observer distance may be variable in time. We explore this possibility in $\S 4$.

In this paper we assume that all of the TNOs are at a single, unknown distance which is then constrained by observations. Of course, TNOs can have quite different eccentricities and semimajor axes so at a given time there will be a radial distribution of TNOs. Since both the geometric covering fraction and TNO temperature decrease with increasing distance from the Sun, we are most sensitive to the closest TNOs. This helps to justify our assumption of a single distance. Moreover, the TNO temperature varies with distance from the Sun, so the radial distribution of TNOs will produce a more complicate spectral distortion than

\footnotetext{
5 There should be no measurable polarization signal as the mean CMB and the TNO emission are both blackbody radiation. Any polarization signal produced by features on the surface of the TNOs will vanish, because the experiment's beam will contain many TNOs that are randomly oriented with respect to one another. If the TNOs are aspherical and have a large magnetic susceptibility, it is possible that they may align with the magnetic field via the Greenstein-Davis effect (Draine 2003). An analysis of this possibility is beyond the scope of this paper.
}

implied by equation (1). With good enough data, this radial distribution could be constrained by constraining the distribution of TNO temperatures. However, with the limited sensitivity of current data our assumption of a single distance is sufficiently accurate. In $\S 4$ we slightly relax this assumption by allowing the TNO distances to be anisotropic; therefore, all of the TNOs in a given pixel will be at the same distance, which will be different than the TNO distance in other pixels. The size distribution of TNOs is specified by their mass function, $n(M)$, with total mass

$$
M_{\mathrm{tot}}=\int n(M) M d M .
$$

For Kuiper Belt objects, the heating is almost entirely solar, so we can calculate the equilibrium temperature of these objects. In the case of Oort Cloud objects, their equilibrium temperature even at large distances is higher than that of the $\mathrm{CMB}$, even though at $25,000 \mathrm{AU}$ the solar contribution is tiny. Interstellar processes, as well as the absorption of CMB photons, results in a floor for the TNO temperature. The equilibrium temperature of Oort Cloud objects is assumed not to drop below 5-6 K, as claimed by Mumma et al. (1993) and Stern (2003). In this work we calculate the temperature of TNOs by assuming thermal equilibrium with the Sun. As mentioned above, this assumption breaks down for TNOs with extremely large semimajor axes ( $a \geq 10,000 \mathrm{AU})$, but objects at such a large distance will produce a signal well below current instrumental sensitivities and therefore are irrelevant for our work. Assuming thermal equilibrium with the Sun, the temperature of a TNO is

$$
T_{\mathrm{TNO}}=\left[\frac{R_{\odot}^{2}}{4 D^{2}}(1-A)\right]^{1 / 4} T_{\odot}
$$

where $R_{\odot}$ is the Sun's radius, $T_{\odot}$ is the temperature of the Sun's photosphere, and $A$ is the TNO's albedo. At distances appropriate for the Kuiper Belt, $D=40 \mathrm{AU}$, we find a temperature of $T=43 \mathrm{~K}$ and at inner Oort Cloud distances, $D=1000 \mathrm{AU}$, the temperature is $T=9 \mathrm{~K}$.

The total distortion will depend on the following parameters of the Oort Cloud and Kuiper Belt. Here we describe our choices for these parameters, as well as theoretical and observational constraints.

Density.-We assume a density $\rho=1 \mathrm{~g} \mathrm{~cm}^{-3}$. This number depends on the porosity of the TNOs and may vary by a factor of a few.

Albedo.-We assume an albedo of $A=4 \%$ as has been measured for comets and is relevant for dirty ice (Luu \& Jewitt 2002). The constraints only depend on the albedo as $(1-A)^{1 / 4}$ so this uncertainty does not strongly affect our results.

Mass function.-We assume a broken power law ${ }^{6}$

$$
\begin{gathered}
n(M)=A M^{-\alpha}, \quad M_{\min }<M<M_{\mathrm{br}}, \\
n(M)=A M_{\mathrm{br}}^{-\alpha+\beta} M^{-\beta}, \quad M_{\mathrm{br}}<M<M_{\max } .
\end{gathered}
$$

The appropriate power-law exponents are not well known. We find that our results are quite sensitive to $\alpha$, the low-mass slope, because for a given total amount of mass in either the Oort Cloud or Kuiper Belt a steeper mass function will increase the geometric

\footnotetext{
6 Typically, the differential mass function is expressed in terms of comet radius, not mass. The power law with respect to radius is related to the values used in this paper as $\alpha_{\text {mass }}=\left(\alpha_{\text {rad }}+2\right) / 3$. The canonical faint-end power-law exponent of $\alpha_{\text {rad }}=3.5$ implies $\alpha_{\text {mass }}=1.83$.
} 
covering fraction. It is difficult to observationally constrain the mass function of comets due to the uncertainties in the dynamics of comet's comae. Collisional equilibrium, along with the assumption that the strength of the object is independent of size, would lead us to expect $\alpha=11 / 6$ (Pan \& Sari 2005). If the larger objects are more difficult to shatter, as would occur if the gravitationally binding energy were very important, then $\alpha<11 / 6$. Since there is so much uncertainty in $\alpha$, we present results for several values. The value of $\beta$ has little effect on our final results if $\beta>2$. We adopt a single value of $\beta=13 / 6$, which is consistent with the current observational results (Bernstein et al. 2004; Pan \& Sari 2005). In $\S 5$ we discuss how our conclusions depend on this assumption.

Lower mass.- The minimum size of surviving objects is determined by the ability of Robertson-Poynting drag to eliminate the smallest objects as well as the properties of collisions that can fragment larger objects into smaller ones (Burns et al. 1979). We take this lower mass to correspond to objects of radius $1 \mathrm{~mm}$. This quantity depends on the detailed formation history of the Kuiper Belt and the Oort Cloud and is difficult to calculate. This quantity is partially degenerate with the total mass of the outer solar system, which is also unknown, so for the purposes of this paper we fix it at $1 \mathrm{~mm}$.

However, we should note that the efficiency of blackbody emission is suppressed when the radiation wavelength is larger than the size of the object emitting the radiation due to Kirchoff's law (Greenberg 1978). This suppression of the TNO emissivity, which will change the low-frequency portion of the CMB spectrum, may be used to constrain the minimum mass. We discuss this in more detail in $\S 3$ where we mention a new proposed $\mathrm{CMB}$ experiment to measure these low frequencies.

Upper mass.-We assume $M_{\max }=10^{-2} M_{\text {Earth }}=6 \times 10^{25} \mathrm{~g}$ as the upper mass. Our results are insensitive to changes of even many orders of magnitude in the upper mass limit for $\beta>2$. If $\beta<2$, a significant fraction of the mass would be at the highmass end and our limits on the low-mass end would not directly translate to limits on the overall mass in the outer solar system.

Break mass.-We assume $M_{\mathrm{br}}=3.2 \times 10^{19} \mathrm{~g}$ (corresponding to $\left.R_{\mathrm{br}}=2 \times 10^{6} \mathrm{~cm}\right)$. The break mass is analytically calculated by determining the largest TNOs that can be in collisional equilibrium over the lifetime of the solar system (Pan \& Sari 2005). Like the power-law indices, this parameter is also uncertain, but like the upper mass, our results are relatively insensitive to its value. Observations with the Hubble Space Telescope have constrained the break radius to be $R_{\mathrm{br}} \leq 20 \mathrm{~km}$ (Bernstein et al. 2004).

Total mass. - The total mass $M_{\mathrm{tot}}$ is one of the things which we seek to constrain with this calculation. Theoretically, the total mass should be (0.1-100) $M_{\text {Earth }}$ (cf. Stern \& Weissman 2001), depending on the surface mass density of the protoplanetary disk and the details of the growth of the planets.

Distance.-We consider what limits can be set for distances from $D=40$ to $10,000 \mathrm{AU}$. The strength of the effect roughly scales as $D^{-4}$, so we are only sensitive to the inner Oort Cloud and not the outer Oort Cloud from where LPCs are observed to originate.

\subsection{Strategies}

In this subsection we outline the two strategies that can be used to constrain the mass distribution in the outer solar system via their spectral distortions to the CMB. For small optical depth $\tau$, the observed intensity fluctuation in the CMB toward a given direction $\hat{\boldsymbol{n}}$ is

$$
\begin{aligned}
I_{\nu}(\hat{\boldsymbol{n}})= & {[1-\tau(\hat{\boldsymbol{n}})]\left[B_{\nu}\left(\bar{T}_{\mathrm{CMB}}+\Delta T(\hat{\boldsymbol{n}})\right)\right] } \\
& +\hat{\boldsymbol{n}} \cdot \boldsymbol{v}+\tau(\hat{\boldsymbol{n}}) B_{\nu}\left(T_{\text {Oort }}(\hat{\boldsymbol{n}})\right)+N_{\mathrm{inst}}
\end{aligned}
$$

corresponding to the extragalactic CMB, including the temperature anisotropy $[\Delta T(\hat{\boldsymbol{n}})]$, extinguished by the TNOs in the beam; the Doppler effect, assuming that the observer has velocity $\boldsymbol{v}$ with respect to the CMB rest frame; TNO blackbody emission; and instrument noise, which is statistically stationary but random. In what follows we assume that the anisotropy produced via the Doppler effect, while in principle large, is calculable and therefore does not affect our ability to extract or constrain the TNO component of the signal. Subtracting off the mean CMB blackbody, the intensity fluctuation in a given direction is

$$
\begin{aligned}
\delta I_{\nu}(\hat{\boldsymbol{n}})= & \frac{\partial B_{\nu}\left(\bar{T}_{\mathrm{CMB}}\right)}{\partial T} \Delta T(\hat{\boldsymbol{n}})+\tau(\hat{\boldsymbol{n}}) \\
& \times\left[B_{\nu}\left(T_{\text {Oort }}(\hat{\boldsymbol{n}})\right)-B_{\nu}\left(\bar{T}_{\mathrm{CMB}}+\Delta T(\hat{\boldsymbol{n}})\right)\right]+N_{\text {inst }},
\end{aligned}
$$

Unfortunately for our purposes, the CMB has small statistical temperature fluctuations ${ }^{7}$ on the order of 1 part in $10^{5}$. We would like to constrain the TNO optical depth, $\tau(\hat{\boldsymbol{n}})$, in a given direction. This is complicated by the random terms in equation (6) corresponding to instrument noise and CMB temperature anisotropies.

We should be able to directly distinguish the $\geq 5 \mathrm{~K}$ blackbody spectrum from the CMB blackbody spectrum, especially when we include data at higher frequencies $(\nu>200 \mathrm{GHz})$, at a level set by either the CMB temperature anisotropies or instrument noise. This would enable us to directly make a map of the TNO mass density. If the instrument pixel noise dominates both the intrinsic variation due to the $\mathrm{CMB}$ temperature anisotropies and signal from the outer solar system, then the constructed TNO map would have a signal-to-noise ratio of less than one and would be useless. In this case, we can reduce the instrument noise by smoothing the $\mathrm{CMB}$ map and averaging together neighboring pixels; this will increase the pixel signal-to-noise ratio. The extreme limit of the procedure is to calculate the mean $\mathrm{CMB}$ spectrum. If the CMB temperature anisotropies dominate the instrument noise, it is possible to reduce, but not completely eliminate, the variance of the temperature anisotropies. This technique is based on differencing the observations of a given position of the celestial sphere made at different locations within the solar system. It is discussed in detail in $\S 4$. Using this technique, it may be possible to make a two- or even three-dimensional map of the distribution of TNOs.

\section{ISOTROPIC SPECTRAL DISTORTION}

\subsection{Observational Constraints}

We use data from FIRAS on the Cosmic Background Explorer (COBE; Fixsen et al. 1997) to place limits on deviations from a blackbody spectrum due to TNOs. FIRAS measured the mean, or monopole, CMB frequency spectrum, so we average the signal originating in the outer solar system over the entire sky in order to compare with their results. The Oort Cloud is assumed to be isotropic, since the observed distribution of inclination angles of new LPCs is approximately uniform (Marsden \& Sekanina 1971), although the Galactic tide (Heisler \& Tremaine 1986) and stellar perturbations (Babich \& Loeb 2007) should make it aspherical.

The Kuiper Belt is highly anisotropic, since it is primarily located near the ecliptic plane. The FIRAS results we use have been averaged over the full sky in each frequency band, and therefore, the signal from the Kuiper Belt must also be averaged

\footnotetext{
7 This implies that it is impossible to predict the exact pattern of observed temperature anisotropies. We can only predict the two-point correlation function (the scale-dependent variance) which parameterizes the probability distribution function of which the observed CMB is a random realization.
} 


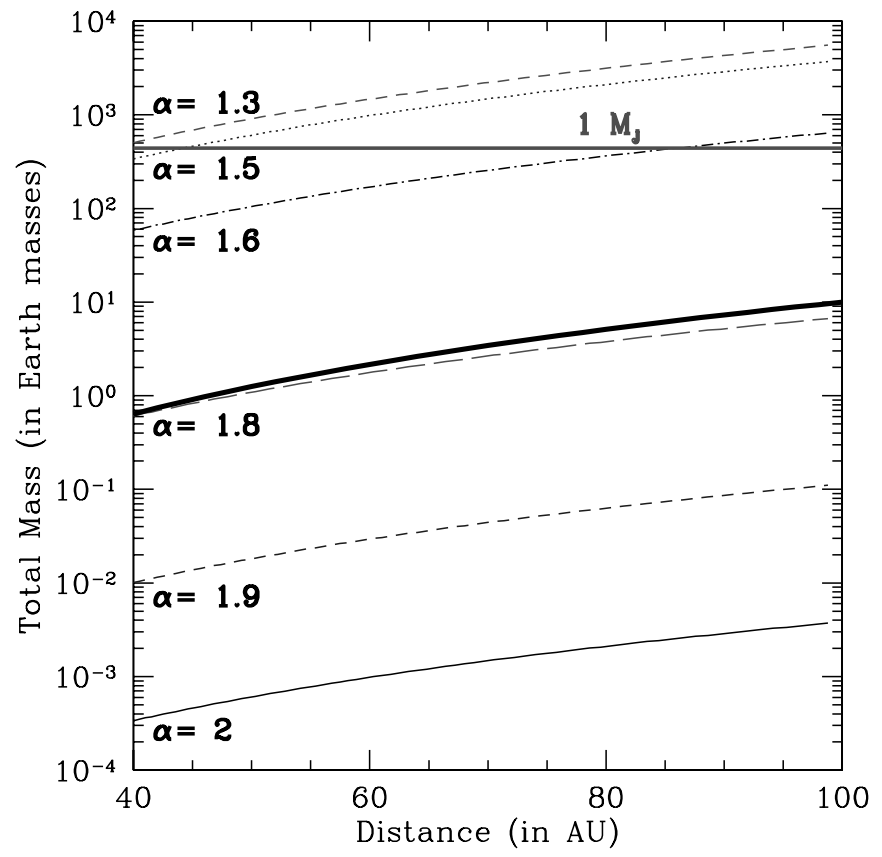

FIG. 1.-Excluded regions of total mass and distance for the Kuiper Belt for different mass function slopes $\alpha$. The curves correspond to $\alpha=2$ (solid line), 1.9 (dashed line), 1.8 (long-dashed line), 1.667 (dot-dashed line), 1.5 (dotted line), and 1.333 (dashed line). The region above the curves is excluded at $95 \%$ by the $C O B E$ FIRAS data. Also shown is a dynamical limit determined by constraints on perturbations to the orbit of Halley's comet (thick solid line) and, for reference, a line corresponding to a Jupiter mass (thick horizontal line). The power law with respect to radius is related to the values used in this paper as $\alpha_{\text {mass }}=\left(\alpha_{\mathrm{rad}}+2\right) / 3$. [See the electronic edition of the Journal for a color version of this figure.]

over the full sky. Since we know that the Kuiper Belt is located at small inclination, it is less optimal to use the full-sky-averaged FIRAS spectrum. This will still allow us to place constraints on the Kuiper Belt, even if they are not the best possible.

Since FIRAS did not detect any spectral distortion in the CMB, we can only place upper limits on the basic parameters of the outer solar system. There are a multitude of processes that can produce spectral distortions, but we ignore them as we are simply using the lack of detection to constrain the properties of the outer solar system. Note that the inclusion of these other effects would only make our upper limits stronger.

Figures 1 and 2 display constraints on the total mass ${ }^{8}$ and the distance of the Kuiper Belt and inner Oort Cloud, respectively, from a nondetection of CMB spectral distortions in the FIRAS data. The confidence contours are determined by calculating the change in the $\chi^{2}$ of the FIRAS data when a component originating from the outer solar system is included. The errors used to calculate the $\chi^{2}$ are taken from Table 4 of Fixsen et al. (1996) for the lowfrequency FIRAS data which extends up to $630 \mathrm{GHz}$.

The curves are shown for several values of $\alpha$, since the constraints strongly depend on the low-mass end of the planetesimal

\footnotetext{
${ }^{8}$ Our technique is mainly sensitive to low-mass objects, so we must be very cautious when interpreting these results. First of all, we are assuming that the mass function is well modeled as a broken power law over a very wide range in mass. Second, Poisson fluctuations become important at the high-mass end, where the expected number of objects, as extrapolated from the mass function, approaches unity. In addition, runaway growth during the core accretion phase of formation can grow a single object to a much larger mass than any other object in its vicinity. A simple extrapolation of the mass function will miss these objects. These last two effects can cause the actual mass of the Kuiper Belt or Oort Cloud to differ significantly from our constraints. Fortunately, these high-mass objects are more easily detected in optical surveys, and so our technique is complementary to existing methods.
}

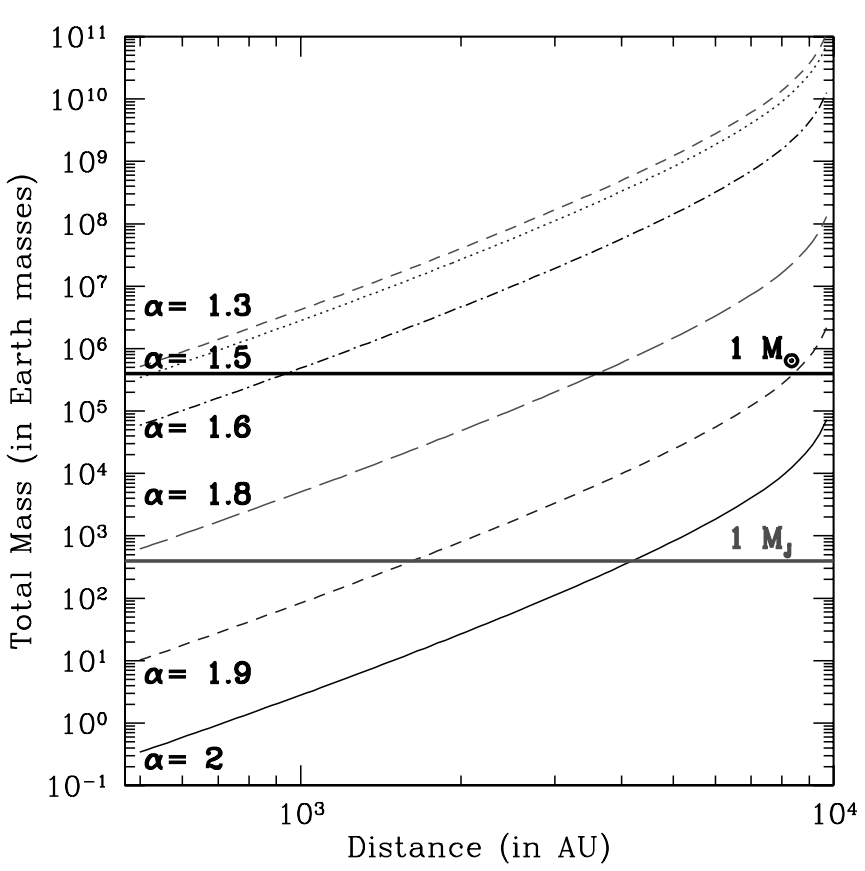

FIG. 2.- Same as Fig. 1, but for the inner Oort Cloud. The region to the upper left of the curves is excluded at $95 \%$ by the COBE FIRAS data. For reference we also include lines corresponding to a solar mass (thick horizontal line) and a Jupiter mass (thick horizontal line). [See the electronic edition of the Journal for a color version of this figure.]

mass function. Even though the Kuiper Belt primarily lies in the ecliptic plane and the FIRAS data is the mean CMB spectrum, averaged over the entire sky, we can still use this data to constrain, in a suboptimal manner, the properties of the Kuiper Belt. These constraints correspond to $95 \%$ confidence limits. Also shown is a dynamical constraint on the total mass in the Kuiper Belt from constraints on the orbit of Halley's comet (Hogg et al. 1991; Hamid et al. 1968). These figures demonstrate that our technique is competitive with dynamical limits on the Kuiper Belt mass and may place more stringent constraints if the slope of the low-mass end of the mass function is steep enough. Hence, if $\alpha_{\text {mass }}=3.5\left(\alpha_{\text {rad }}=1.83\right)$, then the dynamical CMB results place limits to the TNO mass that are as tight as the dynamical limits from Halley's comet's orbit and exclude $M>1 M_{\odot}$ in general. This technique may be the only way we can constrain the properties of the inner Oort Cloud. Of course, our technique is only sensitive to the low-mass end of the distribution and so care must be taken when interpreting these results.

\subsection{Low-Frequency Observations}

A new experiment, the Absolute Radiometer for Cosmology Astrophysics and Diffuse Emission (ARCADE), has been proposed to constrain low-frequency CMB spectral distortions (Kogut et al. 2006). This experiment would measure the CMB spectrum over the full sky in narrow bands between 3 and $30 \mathrm{GHz}$. The addition of these lower frequencies to the COBE FIRAS data would significantly help in constraining the mean Compton $y$-parameter and chemical potential. If the TNOs emit as perfect blackbodies, the low-frequency data would not help us constrain objects in the outer solar system. At these low frequencies, where both the TNO emission spectrum and the CMB spectrum are both in the RayleighJeans limit, we would simply measure a spectrum also in the Rayleigh-Jeans limit at the composite temperature

$$
T=(1-\tau) T_{\mathrm{CMB}}+\tau T_{\mathrm{TNO}} .
$$


Since the CMB temperature is a priori unknown, it is not possible to exclude the contamination of another blackbody at a different temperature using only low-frequency observations. Measurements in the Wien portion of the spectrum allow us to constrain the properties of the outer solar system, since at these relatively high frequencies, the CMB and TNO spectra are significantly different. However, for small enough particles or low enough frequencies, the TNO emissivity will deviate from a perfect blackbody (Greenberg 1978), the emitted spectrum will not be well described by the Rayleigh-Jeans formula, and the low-frequency data will become useful.

The emissivity, $j(\nu)$, of a body in thermal equilibrium at temperature $T$ is determined by Kirchoff's law (Rybicki \& Lightman 1979)

$$
j(\nu)=\alpha(\nu) B_{\nu}(T),
$$

where $\alpha(\nu)$ is the absorptivity and $B_{\nu}(T)$ is the Planck function. In the geometric optics limit, which we employed in $\S 3.1$, the absorptivity is simply determined by the frequency-independent geometric cross section. When the particle size becomes comparable to the radiation's wavelength and the geometric optics limit becomes invalid, the absorptivity becomes frequency-dependent (Spitzer 1978). These deviations may be used to constrain the minimum mass of TNOs.

\section{ANISOTROPIC DISTANCE MODULATION}

In $\S 3$ we considered the spectral distortion of the mean CMB when averaged over a long time period. Implicit in that analysis was the assumption that the Sun-TNO distance $(\bar{D})$ and observerTNO distance $(D)$ are identical and constant. The Sun-TNO distance, $\bar{D}$, which determines the TNO's temperature, is constant during the lifetime of observation, since the relevant periods are approximately $T \sim 300$ yr for the Kuiper Belt and $T \sim$ 31,000 yr for the inner Oort Cloud. The observer-TNO distance $D$ would change during the course of the observations if the satellite looks at the same point on the celestial sphere from different locations within the solar system. This depends on the detailed scan pattern of the relevant experiment. WMAP does view the same position on the celestial sphere from different positions, but Planck will not.

If the scan pattern of the relevant experiment is such that it does observe the same point of the celestial sphere from multiple distances, then the time-independent extragalactic CMB signal can be partially removed and the component arising from the TNO can be better constrained. In this section we study this technique by considering the various time-varying signals and calculating potential constraints that could be produced.

\subsection{Distance-dependent Signal}

The observed intensity fluctuation in the CMB toward a given direction $\hat{\boldsymbol{n}}$, equation (5), is dependent on the TNO-observer distance as

$$
\begin{aligned}
& I_{\nu}(\hat{\boldsymbol{n}}, D)=[1-\tau(\hat{\boldsymbol{n}}, D)]\left[B_{\nu}\left(\bar{T}_{\mathrm{CMB}}+\Delta T(\hat{\boldsymbol{n}})\right)\right] \\
& +\hat{\boldsymbol{n}} \cdot \boldsymbol{v}(D)+\tau(\hat{\boldsymbol{n}}, D) B_{\nu}\left(T 1_{\mathrm{TNO}}(\hat{\boldsymbol{n}}), \bar{D}\right)+N_{\mathrm{inst}}
\end{aligned}
$$

Attempting to pick out the Oort Cloud blackbody directly is difficult due to the statistical nature of the temperature anisotropies, especially if we are restricted to low-frequency data. However, by looking at the same position on the celestial sphere from different points in the CMB experiment's orbit, we can alter the distance between the observer and the TNO. This difference is

$$
\begin{aligned}
& I_{\nu}\left(\hat{\boldsymbol{n}}, D_{i}\right)-I_{\nu}\left(\hat{\boldsymbol{n}}, D_{j}\right)=\left[\tau\left(\hat{\boldsymbol{n}}, D_{i}\right)-\tau\left(\hat{\boldsymbol{n}}, D_{j}\right)\right] \\
& \times\left[B_{\nu}\left(T_{\mathrm{TNO}}(\hat{\boldsymbol{n}}), \bar{D}\right)-B_{\nu}\left(\bar{T}_{\mathrm{CMB}}+\Delta T(\hat{\boldsymbol{n}})\right)\right] \\
& +\hat{\boldsymbol{n}} \cdot\left[\boldsymbol{v}\left(D_{i}\right)-\boldsymbol{v}\left(D_{j}\right)\right]+N_{\text {inst }}^{i}-N_{\text {inst }}^{j} .
\end{aligned}
$$

By taking this difference we have reduced the importance of the portion of the signal $\left[\Delta T \partial B_{\nu}\left(\bar{T}_{\mathrm{CMB}}\right) / \partial T\right]$ that is statistically unknown. Other terms are assumed to be known to such a precision that they can be cleanly removed. So, we consider each change in the intensity fluctuation accompanying a change in the CMB, as our detection limits for the TNO are set by the largest accompanying change.

\subsubsection{Constancy of the $C M B$}

The CMB anisotropies as measured from two different locations are not necessarily identical. There are three effects that potentially could cause small changes: (1) each set of anisotropies have slightly different surfaces of last scattering and therefore depend on slightly different initial curvature perturbations; (2) the path difference between the two different observation points can induce phase shifts in the anisotropy Fourier coefficients, $a_{l m}$, due to free streaming of the anisotropies; and (3) a different ordinary Sachs-Wolfe effect due to different values of the solar system gravitational potential at these different points. The first two effects produce very small changes and are negligible. Note that the ordinary Sachs-Wolfe effect produced by the Sun can be large,

$$
\frac{\Delta T}{\bar{T}_{\mathrm{CMB}}}=\frac{2 G M_{\odot}}{c^{2}(1 \mathrm{AU})} \sim 1.89 \times 10^{-8} ;
$$

however, the uncertainty introduced by the effect is suppressed by the fractional uncertainty in the distance between the CMB experiment and the Sun. The constancy of the CMB anisotropies only allows us to reduce the influence of the anisotropies and their statistical uncertainty from $O(\Delta T)$ to $O(\tau \Delta T)$, not completely eliminate them.

\subsubsection{Doppler Effect}

The relative velocity of the observer with respect to the CMB rest frame induces anisotropies into the observed CMB. For small velocities, the Doppler effect can be decomposed into contributions from the motion of the solar system with respect to the CMB rest frame and the motion of the observer around the Sun. ${ }^{9}$ The first velocity, the motion of the Sun with respect to the CMB rest frame, will be constant on the timescale of observations. As we move to a different position in the Earth's orbit, the Doppler contribution $\hat{\boldsymbol{n}} \cdot \boldsymbol{v}$ will also change, and the two Doppler effect terms in equation (10) will not in general cancel. Even though the anisotropy produced by the Doppler effect is large, its uncertainty is not. The uncertainty in the Earth's orbit is of order $10^{-11}$ in position and velocity, and the uncertainty in the Doppler effect contribution will be of the same order (Standish 2005).

\subsubsection{TNOS}

The temperature of a given TNO is unknown (since the distance to the object is unknown), but is assumed to be constant during the period of CMB observations. The fraction of our beam filled by

\footnotetext{
9 The motion of the observer around the Sun will also induce a Doppler shift in the radiation emitted by the TNOs. This effect is smaller than the Doppler shift of the extragalactic CMB by a factor of the TNO optical depth, $\tau$.
} 
TNOs depends on the variable observer-TNO distance. For all reasonable choices of Kuiper Belt and inner Oort Cloud parameters, the beam is sparsely filled, and therefore, the TNO filling fraction will vary as $D^{-2}$. 10

Some fraction of the TNOs in our beam might be shifted out of the beam due to the parallax effect (especially when we are considering the much closer Kuiper Belt) as the CMB experiment changes its position within the solar system. The fractional change of the beam filling fraction due to the parallax is

$$
f=\frac{2 \pi \theta_{B} \Delta \theta}{\pi \theta_{B}^{2}},
$$

where $\theta_{B}$ is the beam width and $\Delta \theta=(2 \mathrm{AU}) / D$ is the maximum parallax angle in the small-angle approximation. The need to minimize this fractional change will determine the resolution of the TNO map that we can ultimately reconstruct. By requiring this fractional change to be less than $1 \%$, we find

$$
\theta_{B} \geq 200 \frac{1 \mathrm{AU}}{D} .
$$

For the Inner Oort Cloud $\left(D \approx 10^{3} \mathrm{AU}\right) \theta_{B} \geq 10^{\prime}$, and for the Kuiper Belt it is much larger.

In principle we can circumvent this parallax problem and construct maps with resolution comparable to the beam width of the experiment by assuming that the motion of the TNO on the celestial sphere is completely due to the motion of the experiment and then accounting for the shift between the extragalactic CMB and the TNOs. This is extremely complicated but possible for low instrument noise and enough interlocking observations. The details will be presented in a future paper.

When we change the distance to a group of TNOs from $D$ to $D+x$, the fractional change in the TNO contribution to the observed CMB intensity is

$$
\frac{\delta I}{I}=\frac{D}{2 x+x^{2} / D} .
$$

The first-order term is a good approximation for $D \gg x$, but for shorter distances, the second-order term will also contribute. For $D \gg x$ we are able to produce a two-dimensional map of the optical depth from small objects using this method, but for distances such that the second-order term is significant, we are also able to determine the correct distances to these objects and produce a three-dimensional map. The number of TNOs in the beam, which is also unknown, is completely degenerate with the distance $D$ when $D \gg x$, and it is the second-order variation that breaks this degeneracy and allows us to reconstruct both the beam filling fraction and the distance to the TNOs. We consider the potential of this effect to constrain TNO properties in $\S 4.2$.

\subsubsection{Instrument Noise}

Each pixel will have a fluctuation due to instrument noise, since the system temperature is above the CMB temperature. For reference we describe instrument noise in temperature units $\left(\sigma_{T}\right)$ and relate it to uncertainty in the observed intensity as ( Tegmark \& Efstathiou 1996)

$$
\sigma_{B}(\nu)=\frac{\partial B_{\nu}\left(\bar{T}_{\mathrm{CMB}}\right)}{\partial T} \sigma_{T}
$$

\footnotetext{
${ }^{10}$ If the beam were completely filled, then the distance modulation would not change the observed surface brightness.
}

\subsection{Potential Constraints}

We now determine how well this technique can produce a map of TNOs for two model experiments, one with WMAP instrumental parameters and one with next-generation parameters. We ignore the Doppler and Sachs-Wolfe effects discussed in $\S 4.1 .1$ and assume that their amplitudes can be determined with sufficient precision that we can correct for them. The Fisher matrix formalism is employed to estimate these potential constraints (see Tegmark et al. 1997 for an overview of the Fisher matrix formalism).

The intensity in a given direction depends on our orbital position because of the portion coming from the outer solar system. We can isolate this distance-dependent piece by differencing the observed $\mathrm{CMB}$ intensities in a given direction as measured at different orbital positions,

$$
\begin{aligned}
& \Delta \bar{I}_{\nu}(\hat{\boldsymbol{n}})=\sum_{i \neq j} I_{\nu}\left(\hat{\boldsymbol{n}}, \boldsymbol{x}_{i}\right)-I_{\nu}\left(\hat{\boldsymbol{n}}, \boldsymbol{x}_{j}\right) \\
& =\sum_{i \neq j}\left[\tau\left(\hat{\boldsymbol{n}}, \boldsymbol{x}_{i}\right)-\tau\left(\hat{\boldsymbol{n}}, \boldsymbol{x}_{j}\right)\right]\left[B_{\nu}\left(\bar{T}_{\mathrm{TNO}}\right)-B_{\nu}\left(\bar{T}_{\mathrm{CMB}}\right)\right],
\end{aligned}
$$

where the optical depth in a given direction is modeled as having an unknown constant amplitude $\tau_{0}$ in addition to the unknown variable distance

$$
\tau(\hat{\boldsymbol{n}}, \boldsymbol{x})=\frac{\tau_{0}(\hat{\boldsymbol{n}})}{[\bar{D}(\hat{\boldsymbol{n}})+\boldsymbol{x}]^{2}} .
$$

Note that $T_{\mathrm{TNO}}(\hat{\boldsymbol{n}})$ also depends on $\bar{D}(\hat{\boldsymbol{n}})$ through the thermal balance of solar heating, equation (4).

In order to estimate the precision with which we can measure the distance to a TNO we perform a Fisher matrix analysis. The Fisher matrix is defined as

$$
F_{\alpha \beta} \equiv \sum_{\nu} \frac{1}{\sigma_{B}^{2}(\nu)} \frac{\partial \Delta I_{\nu}}{\partial p_{\alpha}} \frac{\partial \Delta I_{\nu}}{\partial p_{\beta}},
$$

where $p_{\alpha}=\left\{\tau_{0}(\hat{\boldsymbol{n}}), \bar{D}(\hat{\boldsymbol{n}})\right\}$. Since $\tau_{0}$ is unknown, we marginalize over this parameter and find the constraints on $\bar{D}$. Uncertainties in the $\mathrm{CMB}$ detector position and velocity, as well as uncertainties in the positions of planets within the solar system, will all increase the overall uncertainty. We expect instrument noise to be the dominant source of uncertainty and assume that the noise in equation (18) solely comes from the instrument noise.

These limits allow us to detect the presence of TNOs in a given direction. To first order in $\Delta x / \bar{D} \sim(1 \mathrm{AU}) / \bar{D}$, we are only sensitive to $\tau_{0} / \bar{D}^{5}$, so our results would be completely degenerate between these two unknown parameters. This degeneracy is broken at second order in $\Delta x / \bar{D}$. We can either try to make a twodimensional map and constrain the TNO optical depth in each pixel or we can use second-order variations in $\Delta x / D$ to make a three-dimensional map and determine both the optical depth in a pixel as well as the mean distance to TNOs in that pixel. Table 1 shows the potential constraints on $\tau_{0}$ and $\bar{D}$ that can be produced by a CMB experiment with a given pixel noise and that observes in 10 frequency bands and measures a given pixel on the sky with 10 different projected distances $-0.5 \mathrm{AU} \leq \Delta x \leq 0.5 \mathrm{AU}$.

The upper portion of Table 1 corresponds to the Kuiper Belt. The plausibility of this technique strongly depends on the value of $\alpha$. Our results imply that a map of the Kuiper Belt can be made if $\alpha \approx 2$. In fact, there is a transitional value of $\alpha_{\text {mass }} \geq 5 / 3$ ( $\alpha_{\text {rad }} \geq 1.22$ ) corresponding to the point where the integral in 
TABLE 1

Detection limits of the optical DePth, $\tau_{0}(\hat{\boldsymbol{n}})$, AND Distance, $\bar{D}(\hat{\boldsymbol{n}})$, For Kuiper Belt and Oort Cloud objects

\begin{tabular}{|c|c|c|c|c|c|}
\hline $\begin{array}{l}\text { Mass } \\
(M)\end{array}$ & $\begin{array}{l}\text { Distance } \\
\text { (AU) }\end{array}$ & $\alpha$ & $\begin{array}{c}\sigma_{T} \\
(\mu \mathrm{K})\end{array}$ & $\sigma_{\tau_{0}} / \tau_{0}$ & $\sigma_{D} / \bar{D}$ \\
\hline 1.................... & 40 & 1.5 & 1 & 19300 & 6470 \\
\hline 1 & 40 & 1.66 & 1 & 3280 & 1100 \\
\hline 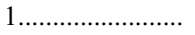 & 40 & 1.8 & 1 & 34.6 & 11.6 \\
\hline 1 & 40 & 1.9 & 1 & 0.575 & 0.193 \\
\hline 1 & 40 & 2 & 1 & 0.0192 & 0.00647 \\
\hline 1 & 40 & 1.5 & 30 & 578000 & 194000 \\
\hline 1 & 40 & 1.66 & 30 & 98500 & 33100 \\
\hline $1 \ldots \ldots \ldots \ldots$ & 40 & 1.8 & 30 & 1040 & 349 \\
\hline 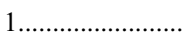 & 40 & 1.9 & 30 & 17.2 & 5.80 \\
\hline 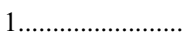 & 40 & 2 & 30 & 0.577 & 0.194 \\
\hline 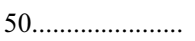 & 1000 & 2 & 1 & 30.0 & 10.0 \\
\hline $50 \ldots \ldots \ldots \ldots \ldots \ldots \ldots$ & 5000 & 2 & 1 & 8700 & 2910 \\
\hline
\end{tabular}

Notes.-The optical depth and distance are partially degenerate, so the uncertainties presented here have marginalized over the second degenerate parameter. In order to make a meaningful map of the outer solar system, the fractional uncertainties must be less than one.

equation (2) becomes dominated by the low-mass end of the distribution. The lower portion of Table 1 corresponds to the inner Oort Cloud. Because of the strong distance dependence of the effect, producing a map of the inner Oort Cloud will be much more challenging. Assuming $\alpha=1.9$ and $\sigma_{T}=1 \mu \mathrm{K}$, there needs to be $M_{\text {tot }}=0.185 M_{\text {Earth }}$ in order to produce a twodimensional map with a signal-to-noise ratio of unity.

\section{DISCUSSION}

In this paper we describe two new techniques to constrain the cumulative mass contained in the outer solar system. The distribution of mass in this portion of the solar system may provide clues to both the early nature of and the formation scenario for the solar system. Current techniques for finding these objects that rely on the detection of reflected sunlight are only sensitive to the largest and closest members of this population, objects that are rare and therefore are likely to be special cases. Our new techniques directly probe the low-mass end of the distribution that is otherwise quite difficult to detect.

It has been suggested that observations of occultation events may be used to constrain the low-mass end of the TNO distribution. Bodies in the Kuiper Belt can be detected when they transit in front of distant luminous objects, resulting in a brief dip in the observed brightness. The Taiwan-America Occultation Survey (Lehner et al. 2006) seeks to conduct a census of large Kuiper Belt objects using background stars. The cadence of observation required to detect the smallest objects presents significant tech- nological challenges because of optical CCD read noise. This same occultation method has been applied to Scorpius X-1, the brightest X-ray source near the ecliptic plane (Chang et al. 2006), since X-ray detectors can be operated at a higher cadence than optical CCDs. However, the small number of bright X-ray sources near the ecliptic limits the total volume of the Kuiper Belt that can be surveyed.

Our method does not suffer from these limitations, as the CMB detector technology is proven and the CMB can be accurately observed over nearly the entire sky (except for the Galactic plane). The main drawback of our proposed techniques is their reliance on the unknown TNO mass function. We must also be careful when interpreting our results, because we are basically constraining the low-mass end of the TNO distribution and then using those results to infer the total mass in the outer solar system. If the slope of the high-mass end of the mass function is steep $(\beta>2)$, then most of the mass lies in the low-mass end and this inference is valid. However, if the slope is relatively shallow $(\beta<2)$, then we should only interpret our results as pertaining to the mass in small objects. Current observational results allow for both possibilities (Bernstein et al. 2004), and hopefully, future observations will determine the true slope. These concerns are in addition to those expressed in $\S 3$ regarding the possibility that rare high-mass objects could cause the true total mass to differ from that inferred by extrapolating the mass function. Fortunately, the large objects are the easiest to optically detect, and our proposed techniques can work together with these surveys to provide us with an accurate description of the outer solar system.

The next stage is to use the data provided by WMAP and analyze it as discussed in $\S 4$ in order to produce a map of Kuiper Belt objects. The data analysis, which must be done with the timeordered data, is a lengthy process, which is why it was not included in this paper. The potential to better understand the formation of our solar system, and subsequently other planetary systems as well, will certainly justify the effort.

The authors would like to thank an anonymous referee whose helpful comments improved the quality of this manuscript. We would like to thank C. Alcock, M. Brown, D. Finkbeiner, D. Fixsen, P. Goldreich, C. Hirata, M. Holman, S. Kenyon, A. Loeb, L. Page, M. Pan, G. Rybicki, R. Sari, D. Spergel, and P. Thaddeus for helpful conversations. D. B. thanks the hospitality of the Harvard Institute for Theory and Computation where some of this work was completed and acknowledges financial support from the Betty and Gordon Moore Foundation. C. L. S. was supported under the National Science Foundation Graduate Research Fellowship Program. C. H. B. acknowledges support from the Harvard Origins of Life Initiative.
Babich, D., \& Loeb, A. 2007, MNRAS, submitted

Backman, D. E., Dasgupta, A., \& Stencel, R. E. 1995, ApJ, 450, L35

Bernstein, G. M., et al. 2004, AJ, 128, 1364

Brown, M. E., Trujillo, C. A., \& Rabinowitz, D. L. 2004, ApJ, 617, 645 2005, ApJ, 635, L97

Burns, J. A., Lamy, P. L., \& Soter, S. 1979, Icarus, 40, 1

Chang, H.-K., et al. 2006, Nature, 442, 660

Dones, L., et al. 2004, in ASP Conf. Ser. 323, Star Formation in the Interstellar

Medium, ed. D. Johnstone et al. (San Francisco: ASP), 371

Draine, B. T. 2003, ARA\&A, 41, 241

Fernandez, J. A. 1997, Icarus, 129, 106

Fixsen, D. J., \& Dwek, E. 2002, ApJ, 578, 1009

Fixsen, D. J., Hinshaw, G., Bennett, C. L., \& Mather, J. C. 1997, ApJ, 486, 623

Fixsen, D. J., \& Kogut, A. 2002, ApJ, 581, 817

\section{REFERENCES}

Fixsen, D. J., et al. 1996, ApJ, 473, 576

Goldreich, P., Lithwick, Y., \& Sari, R. 2004, ARA\&A, 42, 549

Greenberg, J. M., 1978, in Cosmic Dust (New York: Wiley), 187

Hamid, S. E., Marsden, B. G., \& Whipple, F. L. 1968, AJ, 73, 727

Heisler, J., \& Tremaine, S. 1986, Icarus, 65, 13

Hills, J. G. 1981, AJ, 86, 1730

Hogg, D. W., Quinlan, G. D., \& Tremaine, S. 1991, AJ, 101, 2274

Kenyon, S. J., \& Windhorst, R. A. 2001, ApJ, 547, L69

Kogut, A., et al 2006, NewA Rev., 50, 925

Lehner, M. J., et al. 2006, Astron. Nachr., 327, 814

Lissauer, J., \& Stevenson, D. 2007, in Protostars and Planets V, ed. B. Reipurth,

D. Jewitt, \& K. Keil (Tucson: Univ. Arizona Press), 591

Luu, J. X., \& Jewitt, D. C. 2002, ARA\&A, 40, 63

Marsden, B. G., \& Sekanina, Z. 1971, AJ, 76, 1135 
Mather, J. C., et al. 1991, in AIP Conf. Proc. 222, After the First Three Minutes, ed. S. S. Hold, C. L. Bennett, \& V. Trimble (New York: AIP), 43

Mumma, M. J., Weissman, P. R., \& Stern, S. A. 1993, in Protostars and Planets

III, ed. J. I. Lunine \& E. H. Levy (Tucson: Univ. Arizona Press), 1177

Oort, J. H. 1950, Bull. Astron. Inst. Netherlands, 11, 91

Pan, M., \& Sari, R. 2005, Icarus, 173, 342

Peebles, P. J. E. 1993, Principles of Physical Cosmology (Princeton: Princeton Univ. Press), 131

Rybicki, G. B., \& Lightman, A. P. 1979, Radiative Processes in Astrophysics (New York: Wiley), 16
Spitzer, L. 1978, Physical Processes in the Interstellar Medium (New York: Wiley), 157

Standish, E. M. 2005, in IAU Colloq. 196, Transits of Venus, ed. D. W. Kurtz (Cambridge: Cambridge Univ. Press), 163

Stern, A. 2003, Nature, 424, 639

Stern, A., \& Weissman, P. 2001, Nature, 409, 589

Tegmark, M., \& Efstathiou, G. 1996, MNRAS, 281, 1297

Tegmark, M., \& Silk, J 1994, ApJ, 423, 529

Tegmark, M., Taylor, A. N., \& Heavens, A. F. 1997, ApJ, 480, 22

Teplitz, V. L., et al. 1999, ApJ, 516, 425 